\title{
Avaliação comparativa dos efeitos maxilares da expansão rápida da maxila com os aparelhos de Haas e Hyrax
}

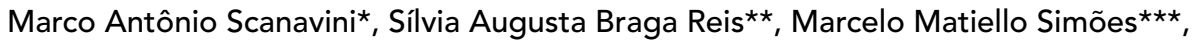

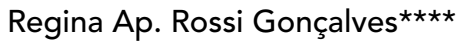

\section{Resumo}

Objetivo: avaliar cefalometricamente os efeitos da expansão rápida da sutura palatina mediana sobre o posicionamento vertical e sagital da maxila, comparando os aparelhos de Haas e Hyrax. Metodologia: a amostra consistiu de 93 telerradiografias obtidas de 31 pacientes jovens, brasileiros, de ambos os gêneros, na faixa etária inicial média de 13 anos e 2 meses. As radiografias foram tomadas ao início do tratamento (pré-disjunção), imediatamente após a disjunção (pós-disjunção) e ao final do nivelamento. Resultados e Conclusões: constatou-se que os dois aparelhos disjuntores apresentaram resultados semelhantes, com a ocorrência de deslocamento da maxila em direção inferior, sem rotação, que se manteve ao final do nivelamento e ocorrência de um deslocamento anterior logo após a disjunção, que retornou aos valores pré-disjunção ao final do nivelamento.

Palavras-chave: Expansão rápida da maxila. Cefalometria. Maxila.

\section{INTRODUÇÃO}

Um dos procedimentos clínicos mais consagrados na prática ortodôntica, por sua eficiência e previsibilidade, é a expansão rápida da maxila ${ }^{11}$ (ERM). A ERM ou disjunção, corrige a atresia transversal da maxila, má oclusão extremamente freqüente, que se estabelece precocemente e não apresenta auto-correção ${ }^{36}$.

A difusão dessa técnica na Ortodontia mundial foi responsabilidade dos estudos e esforços do Prof. Andrew Haas, que desenvolveu o aparelho dentomucosuportado que leva seu nome e estabeleceu o protocolo de disjunção, permitindo o estudo dos resultados e da estabilidade desse procedimento $^{17,18,19,20,21}$.

A disjunção pode ser realizada por meio de aparelhos expansores fixos como o de Haas, que é um aparelho dentomucosuportado, Hyrax e o Disjuntor de McNamara, que são classificados como dentosuportados. Os disjuntores apresentam um parafuso expansor, localizado paralelamente à sutura palatina mediana, ativado de forma a acumular uma quantidade significativa de forças com o objetivo de romper a resistência oferecida pela referida sutura e pelas suturas pterigopalatina, frontomaxilar, nasomaxilar e zigomático-maxilar.

* Coordenador do Curso de Pós-graduação em Odontologia, área de concentração Ortodontia, da Universidade Metodista de São Paulo.

** Mestre em Odontologia, área de concentração Ortodontia, pela Universidade Metodista de São Paulo e Professora Assistente do Departamento de Ortodontia da Universidade Metodista de São Paulo.

*** Mestre em Odontologia, área de concentração Ortodontia, pela Universidade Metodista de São Paulo.

**** Aluna do Curso de Pós-graduação em Odontologia, área de concentração Ortodontia, da Universidade Metodista de São Paulo. 
Devido ao caráter ortopédico do procedimento, o mesmo apresenta limitação de idade para sua realização. Após o final do crescimento ativo, a quantidade de força necessária para o rompimento da sutura fica significativamente alta, resultando em dor, possibilidade de fenestração radicular nos aparelhos dentosuportados ou de necrose da mucosa palatina nos aparelhos dentomucosuportados. Nesses casos, a disjunção é associada a um procedimento cirúrgico, denominado expansão cirurgicamente assistida, que rompe a resistência sutural e permite a disjunção sem os efeitos colaterais já relatados ${ }^{11}$.

Os efeitos da disjunção sobre a maxila e mandíbula, nos sentidos vertical e ântero-posterior, avaliados em telerradiografias de perfil, já foram fartamente investigados ${ }^{6,9,12,14,16,17,18,19,21,22,26,34,42}$. Entretanto, verificamos a necessidade de estudar, comparativamente, os efeitos da disjunção realizada com os aparelhos de Haas e Hyrax sobre a maxila nos sentidos vertical e ântero-posterior.

\section{MATERIAL E MÉTODO}

A amostra utilizada consistiu em 93 telerradiografias de perfil de 31 pacientes jovens, brasileiros, de ambos os gêneros, com idade média de 13 anos e 2 meses no início do tratamento, sendo 16 do gênero masculino e 15 do feminino, com dentição permanente e indicação de disjunção maxilar para correção de atresia maxilar. Os pacientes foram aleatoriamente divididos em dois grupos, cada um tratado com um tipo diferente de disjuntor.

O Grupo I foi composto por 18 pacientes, 9 de cada gênero, com idade média de 13 anos e 6 meses ( 9 anos e 6 meses a 15 anos e 8 meses) no início do tratamento, tratados com o aparelho disjuntor dentomucossuportado (Haas). Os outros treze pacientes constituíram o Grupo II, distribuídos em 7 indivíduos do gênero masculino e 6 do gênero feminino, com idade média de 13 anos e 5 meses ( 11 anos e 9 meses a 15 anos e 2 meses).

Os pacientes foram selecionados entre os que se inscreveram para tratamento ortodôntico no curso de Pós-graduação em Ortodontia da Universidade Metodista de São Paulo (UMESP).

O procedimento clínico da ERM foi padronizado. Incluiu uma fase ativa, iniciada 24 horas após a instalação do aparelho com $1 / 4$ de ativação a cada 12 horas, totalizando $1 / 2$ volta ao dia até a sobrecorreção evidenciada pelo toque da cúspide palatina do primeiro molar superior na cúspide vestibular do molar do primeiro molar inferior. A ativação foi realizada durante 11 a 14 dias, em média, nos dois grupos da amostra.

Após a interrupção da ativação, o parafuso expansor foi estabilizado com resina acrílica, e o aparelho mantido passivamente por 90 dias, até a completa reorganização da sutura palatina mediana. Essa fase foi denominada passiva.

Após a fase passiva, o expansor foi removido e aparelhos removíveis de acrílico foram instalados e mantidos por 6 meses. Logo após a disjunção, os pacientes foram submetidos a tratamento ortodôntico corretivo com aparelho Edgewise para finalização do caso.

Para cada paciente, foram obtidas três telerradiografias laterais padronizadas com os dentes em oclusão cêntrica, sendo estas antes da instalação do aparelho, ao final da fase ativa de expansão e ao final da fase de nivelamento ortodôntico.

As telerradiografias de perfil foram submetidas a traçados cefalométricos manuais das estruturas anátomo-radiológicas de interesse à elaboração do cefalograma, em folhas de acetato ultraphan. As medições lineares foram executadas com um paquímetro com precisão de $0,5 \mathrm{~mm}$ e transferidor com subdivisão de $0,5^{\circ}$.

Para a avaliação da posição ântero-posterior da maxila foram usadas as seguintes variáveis:

- SNA: ângulo formado entre as linhas SN e $\mathrm{NA}$;

- Nperp-A: menor distância entre a linha Nperp e o ponto A;

- CoA: distância entre os pontos Co (condílio) e A (subespinhal).

O deslocamento vertical da maxila foi avaliado 
pelos seguintes fatores:

- ENA-SN: corresponde à menor distância entre o ponto ENA e a linha SN;

- ENP-SN: corresponde à menor distância entre o ponto ENP e a linha SN;

- SN.PP: ângulo formado entre a linha SN e o plano palatino.

O comportamento estatístico das variáveis foi determinado através das médias aritméticas e desvios-padrão para cada variável cefalométrica nas distintas fases em estudo do tratamento, para ambos os grupos.

Foi aplicada a Análise de Variância de Medidas Repetidas, para a verificação das diferenças significantes entre grupos e entre tempos, para cada variável. Quando foram encontradas diferenças entre tempos, grupos ou interação grupo/tempo, foram realizados contrastes para verificar entre quais momentos existiram diferenças estatisticamente significantes.

\section{RESULTADOS}

Os valores obtidos nas mensurações de cada variável cefalométrica, em suas médias aritméticas e desvios-padrão, e a aplicação dos testes estatísticos encontram-se dispostos conforme as tabelas a seguir, tanto para o Grupo I (aparelhos disjuntores dentomucossuportados) quanto para o Grupo II (dentossuportados), nas fases 1) início de tratamento, 2) pós-disjunção e 3) final de nivelamento.

As tabelas 1 e 2 apresentam as médias e os desvios-padrão das medidas cefalométricas analisadas, de ambos os grupos, nas fases 1 (início de tratamento), 2 (pós-disjunção) e 3 (final de nivelamento).

As tabelas 3 a 8 apresentam as médias, os desvios-padrão e as médias das diferenças entre as fases início de tratamento e pós-disjunção (T2-T1); pós-disjunção e final de nivelamento (T3-T2); e início de tratamento e final de nivelamento (T3-T1), em ambos os Grupos - I e II, com aparelhos disjuntores dentomucossuportados e dentossuportados, respectivamente.
As tabelas 9 a 18 demonstram o comportamento estatístico das variáveis, na apresentação da análise de variância de medidas repetidas para as medidas angulares e lineares estudadas, bem como, o resultado dos contrastes para as variáveis estatisticamente significantes.

Tabela 1 - Médias $(\bar{X})$ e desvios-padrão (d.p.) das medidas cefalométricas analisadas no Grupo I (aparelhos disjuntores dentomucossuportados - tipo Haas), nas fases 1 (início de tratamento), 2 (pós-disjunção) e 3 (final de nivelamento).

\begin{tabular}{lccccccc}
\hline \multicolumn{1}{c}{ Fase } & $\begin{array}{c}\text { (1) Início de } \\
\text { tratamento }\end{array}$ & \multicolumn{2}{c}{$\begin{array}{c}\text { (2) Pós-dis- } \\
\text { junção }\end{array}$} & \multicolumn{2}{c}{$\begin{array}{c}\text { (3) Final de } \\
\text { nivelamento }\end{array}$} \\
\hline HAAS & $\begin{array}{c}\mathbf{N} \\
\mathbf{1 8}\end{array}$ & $\overline{\mathbf{X}}$ & d.p. & $\overline{\mathbf{X}}$ & d.p. & $\overline{\mathbf{X}}$ & d.p. \\
\hline Medidas & & & & & & \\
\hline SNA & 79.28 & 3.61 & 79.97 & 3.43 & 79.19 & 3.26 \\
SN.PP & 10.03 & 3.19 & 9.39 & 3.53 & 9.89 & 3.40 \\
ENA-SN & 54.39 & 2.84 & 55.44 & 2.89 & 56.50 & 2.47 \\
ENP-SN & 45.08 & 3.24 & 46.56 & 2.97 & 47.14 & 2.92 \\
Nperp-A & -2.72 & 4.40 & -1.83 & 3.78 & -2.72 & 4.65 \\
Co-A & 88.97 & 4.36 & 90.03 & 4.41 & 90.92 & 4.74 \\
\hline
\end{tabular}

Tabela 2 - Médias $(\bar{X})$ e desvios-padrão (d.p.) das medidas cefalométricas analisadas no Grupo II (aparelhos disjuntores dentossuportados - tipo Hyrax), nas fases 1 (início de tratamento), 2 (pós-disjunção) e 3 (final de nivelamento)

\begin{tabular}{lccccccc}
\hline \multicolumn{1}{c}{ Fase } & \multicolumn{2}{c}{$\begin{array}{c}\text { (1) Início de } \\
\text { tratamento }\end{array}$} & \multicolumn{2}{c}{$\begin{array}{c}\text { (2) Pós-dis- } \\
\text { junção }\end{array}$} & \multicolumn{2}{c}{$\begin{array}{c}\text { (3) Final de } \\
\text { nivelamento }\end{array}$} \\
\hline HYRAX & $\mathbf{N}$ & $\overline{\mathbf{X}}$ & d.p. & $\overline{\mathbf{X}}$ & d.p. & $\overline{\mathbf{X}}$ & d.p. \\
\hline Medidas & & & & & & \\
\hline SNA & 79.62 & 2.37 & 79.96 & 2.18 & 80.31 & 2.62 \\
SN.PP & 9.19 & 3.97 & 9.50 & 3.98 & 9.04 & 4.14 \\
ENA-SN & 54.77 & 3.76 & 55.85 & 3.65 & 56.50 & 4.37 \\
ENP-SN & 46.15 & 3.21 & 47.00 & 3.42 & 47.58 & 4.19 \\
Nperp-A & -1.04 & 3.61 & 0.00 & 3.20 & -0.35 & 3.23 \\
Co-A & 89.88 & 4.75 & 90.92 & 4.70 & 92.38 & 5.52 \\
\hline
\end{tabular}


Tabela 3 - Médias $(\bar{X})$, desvios-padrão (d.p.) e médias das diferenças das medidas cefalométricas analisadas no Grupo I (aparelhos disjuntores dentomucossuportados - tipo Haas), nas fases 1 (início de tratamento) e 2 (pós-disjunção).

\begin{tabular}{lccccccc}
\hline \multicolumn{1}{c}{ Fase } & \multicolumn{2}{c}{$\begin{array}{c}\text { (1) Início de } \\
\text { tratamento }\end{array}$} & \multicolumn{2}{c}{$\begin{array}{c}\text { (2) } \\
\text { Pós-disjunção }\end{array}$} & \multicolumn{2}{c}{$\begin{array}{c}\text { Diferença } \\
(\mathbf{2})\end{array}$} \\
\hline HAAS & $\begin{array}{c}\mathbf{N} \\
\mathbf{1 8}\end{array}$ & $\overline{\mathbf{X}}$ & $\mathbf{d . p .}$ & $\overline{\mathbf{X}}$ & d.p. & $\overline{\mathbf{X}}$ & d.p. \\
\hline Medidas & & & & & & \\
\hline SNA & 79.28 & 3.61 & 79.97 & 3.43 & 0.69 & 0.69 \\
SN.PP & 10.03 & 3.19 & 9.39 & 3.53 & -0.64 & 1.54 \\
ENA-SN & 54.39 & 2.84 & 55.44 & 2.89 & 1.06 & 1.06 \\
ENP-SN & 45.08 & 3.24 & 46.56 & 2.97 & 1.47 & 1.16 \\
Nperp-A & -2.72 & 4.40 & -1.83 & 3.78 & 0.89 & 2.74 \\
Co-A & 88.97 & 4.36 & 90.03 & 4.41 & 1.06 & 1.52 \\
\hline
\end{tabular}

Tabela 5 - Médias $(\bar{X})$, desvios-padrão (d.p.) e médias das diferenças das medidas cefalométricas analisadas no Grupo I (aparelhos disjuntores dentomucossuportados - tipo Haas), nas fases 1 (início de tratamento) e 3 (final de nivelamento).

\begin{tabular}{lccccccc}
\hline \multicolumn{1}{c}{ Fase } & $\begin{array}{c}\text { (1) Início de } \\
\text { tratamento }\end{array}$ & \multicolumn{2}{c}{$\begin{array}{c}\text { (3) Final de } \\
\text { nivelamento }\end{array}$} & \multicolumn{2}{c}{$\begin{array}{c}\text { Diferença } \\
(\mathbf{3})-(\mathbf{1})\end{array}$} \\
\hline HAAS & $\begin{array}{c}\mathbf{N} \\
\mathbf{1 8}\end{array}$ & $\overline{\mathbf{X}}$ & d.p. & $\overline{\mathbf{X}}$ & d.p. & $\overline{\mathbf{X}}$ & d.p. \\
\hline Medidas & & & & & & \\
\hline SNA & 79.28 & 3.61 & 79.19 & 3.26 & -0.08 & 1.27 \\
SN.PP & 10.03 & 3.19 & 9.89 & 3.40 & -0.14 & 1.29 \\
ENA-SN & 54.39 & 2.84 & 56.50 & 2.47 & 2.11 & 2.13 \\
ENP-SN & 45.08 & 3.24 & 47.14 & 2.92 & 2.06 & 1.64 \\
Nperp-A & -2.72 & 4.40 & -2.72 & 4.65 & 0.00 & 2.76 \\
Co-A & 88.97 & 4.36 & 90.92 & 4.74 & 1.94 & 2.18 \\
\hline
\end{tabular}

\section{DISCUSSÃO}

A terapia pela expansão maxilar teve seu início com o então ortodontista Angel1², em 1860, que utilizou um dispositivo com parafuso expansor e observou, clinicamente, o alargamento transversal da maxila. Este fato refletiu o início
Tabela 4 - Médias $(\bar{X})$, desvios-padrão (d.p.) e médias das diferenças das medidas cefalométricas analisadas no Grupo I (aparelhos disjuntores dentomucossuportados - tipo Haas), nas fases 2 (pós-disjunção) e 3 (final de nivelamento).

\begin{tabular}{lccccccc}
\hline \multicolumn{1}{c}{ Fase } & \multicolumn{2}{c}{$\begin{array}{c}\text { (2) Pós-dis- } \\
\text { junção }\end{array}$} & \multicolumn{2}{c}{$\begin{array}{c}\text { (3) Final de } \\
\text { nivelamento }\end{array}$} & \multicolumn{2}{c}{$\begin{array}{c}\text { Diferença } \\
\text { (3) - (2) }\end{array}$} \\
\hline HAAS & $\begin{array}{c}\mathbf{N} \\
\mathbf{1 8}\end{array}$ & $\overline{\mathbf{X}}$ & d.p. & $\overline{\mathbf{X}}$ & d.p. & $\overline{\mathbf{X}}$ & d.p. \\
\hline Medidas & & & & & & \\
\hline SNA & 79.97 & 3.43 & 79.19 & 3.26 & -0.78 & 1.06 \\
SN.PP & 9.39 & 3.53 & 9.89 & 3.40 & 0.50 & 1.06 \\
ENA-SN & 55.44 & 2.89 & 56.50 & 2.47 & 1.06 & 1.83 \\
ENP-SN & 46.56 & 2.97 & 47.14 & 2.92 & 0.58 & 1.48 \\
Nperp-A & -1.83 & 3.78 & -2.72 & 4.65 & -0.89 & 2.54 \\
Co-A & 90.03 & 4.41 & 90.92 & 4.74 & 0.89 & 1.98 \\
\hline
\end{tabular}

Tabela 6 - Médias (X) , desvios-padrão (d.p.) e médias das diferenças das medidas cefalométricas analisadas no Grupo II (aparelhos disjuntores dentossuportados - tipo Hyrax), nas fases 1 (início de tratamento) e 2 (pós-disjunção).

\begin{tabular}{lccccccc}
\hline \multicolumn{1}{c}{ Fase } & $\begin{array}{c}\text { (1) Início de } \\
\text { tratamento }\end{array}$ & \multicolumn{2}{c}{$\begin{array}{c}\text { (2) } \\
\text { Pós-disjunção }\end{array}$} & \multicolumn{2}{c}{$\begin{array}{c}\text { Diferença } \\
\text { (2) }-\mathbf{( 1 )}\end{array}$} \\
\hline HYRAX & $\begin{array}{c}\mathbf{N} \\
\mathbf{1 3}\end{array}$ & $\overline{\mathbf{X}}$ & d.p. & $\overline{\mathbf{X}}$ & d.p. & $\overline{\mathbf{X}}$ & d.p. \\
\hline Medidas & & & & & & \\
\hline SNA & 79.62 & 2.37 & 79.96 & 2.18 & 0.35 & 0.66 \\
SN.PP & 9.19 & 3.97 & 9.50 & 3.98 & 0.31 & 1.28 \\
ENA-SN & 54.77 & 3.76 & 55.85 & 3.65 & 1.08 & 1.02 \\
ENP-SN & 46.15 & 3.21 & 47.00 & 3.42 & 0.85 & 1.33 \\
Nperp-A & -1.04 & 3.61 & 0.00 & 3.20 & 1.04 & 2.44 \\
Co-A & 89.88 & 4.75 & 90.92 & 4.70 & 1.04 & 1.35 \\
\hline
\end{tabular}

de um questionamento polêmico: a possibilidade da abertura da sutura palatina mediana por meio de procedimentos ortodônticos. No entanto, somente na segunda metade deste século, as respostas histológicas, fisiológicas e anatômicas desse procedimento foram pesquisadas, 
Tabela 7 - Médias ( $\bar{X}$, desvios-padrão (d.p.) e médias das diferenças das medidas cefalométricas analisadas no Grupo II (aparelhos disjuntores dentossuportados - tipo Hyrax), nas fases 2 (pós-disjunção) e 3 (final de nivelamento).

\begin{tabular}{|c|c|c|c|c|c|c|c|}
\hline \multicolumn{2}{|c|}{ Fase } & \multicolumn{2}{|c|}{$\begin{array}{l}\text { (2) Pós-dis- } \\
\text { junção }\end{array}$} & \multicolumn{2}{|c|}{$\begin{array}{l}\text { (3) Final de } \\
\text { nivelamento }\end{array}$} & \multicolumn{2}{|c|}{$\begin{array}{c}\text { Diferença } \\
\text { (3) - (2) }\end{array}$} \\
\hline HYRAX & $\begin{array}{l}N \\
13\end{array}$ & $\bar{x}$ & d.p. & $\bar{x}$ & d.p. & $\bar{x}$ & d.p. \\
\hline \multicolumn{8}{|c|}{ Medidas } \\
\hline SN & & 79.96 & 2.18 & 80.31 & 2.62 & 0.35 & 1.59 \\
\hline SN.F & & 9.50 & 3.98 & 9.04 & 4.14 & -0.46 & 1.01 \\
\hline ENA- & & 55.85 & 3.65 & 56.50 & 4.37 & 0.65 & 1.33 \\
\hline ENP- & & 47.00 & 3.42 & 47.58 & 4.19 & 0.58 & 1.37 \\
\hline Nper & & 0.00 & 3.20 & -0.35 & 3.23 & -0.35 & 2.54 \\
\hline Co- & & 90.92 & 4.70 & 92.38 & 5.52 & 1.46 & 2.30 \\
\hline
\end{tabular}

Tabela 9 - Análise de variância de medidas repetidas para a variável SNA.

\begin{tabular}{ccccc}
\hline Variável & $\begin{array}{c}\text { Fonte de variabi- } \\
\text { lidade }\end{array}$ & $\begin{array}{c}\text { Quadrado } \\
\text { Médio }\end{array}$ & F & $\begin{array}{c}\text { Nível de sig- } \\
\text { nificância (p) }\end{array}$ \\
\hline SNA & Grupo & 5.2187 & 0.20 & 0.6601 NS \\
SNA & Tempo & 2.0632 & 2.78 & 0.0908 NS \\
SNA & Grupo x Tempo & 2.4987 & 3.37 & 0.0604 NS \\
\hline
\end{tabular}

NS: não significante, ${ }^{*}$ : significante ao $5 \%(p<0.05),{ }^{* *}$ : significante ao $1 \%(\mathrm{p}<0.01)$

Tabela 11 - Análise de variância de medidas repetidas para a variável SN.PP.

\begin{tabular}{ccccc}
\hline Variável & $\begin{array}{c}\text { Fonte de varia- } \\
\text { bilidade }\end{array}$ & $\begin{array}{c}\text { Quadra- } \\
\text { do Médio }\end{array}$ & F & $\begin{array}{c}\text { Nível de signi- } \\
\text { ficância (p) }\end{array}$ \\
\hline SN.PP & Grupo & 6.2399 & 0.16 & 0.6903 NS \\
SN.PP & Tempo & 0.2477 & 0.29 & 0.7430 NS \\
SN.PP & Grupo x Tempo & 2.2907 & 2.65 & 0.0819 NS \\
\hline
\end{tabular}

NS: não significante, ${ }^{*}$ : significante ao $5 \%(p<0.05),{ }^{* *}$ : significante ao $1 \%(p<0.01)$
Tabela 8 - Médias ( $\bar{X}$ ), desvios-padrão (d.p.) e médias das diferenças das medidas cefalométricas analisadas no Grupo II (aparelhos disjuntores dentossuportados - tipo Hyrax), nas fases 1 (início de tratamento) e 3 (final de nivelamento).

\begin{tabular}{|c|c|c|c|c|c|c|c|}
\hline \multicolumn{2}{|c|}{ Fase } & \multicolumn{2}{|c|}{$\begin{array}{l}\text { (1) Início de } \\
\text { tratamento }\end{array}$} & \multicolumn{2}{|c|}{$\begin{array}{l}\text { (3) Final de } \\
\text { nivelamento }\end{array}$} & \multicolumn{2}{|c|}{$\begin{array}{l}\text { Diferença } \\
\text { (3) - (1) }\end{array}$} \\
\hline HYRAX & $\begin{array}{l}N \\
13\end{array}$ & $\overline{\mathbf{x}}$ & d.p. & $\overline{\mathbf{x}}$ & d.p. & $\bar{x}$ & d.p. \\
\hline \multicolumn{8}{|c|}{ Medidas } \\
\hline \multicolumn{2}{|c|}{ SNA } & 79.62 & 2.37 & 80.31 & 2.62 & 0.69 & 1.80 \\
\hline \multicolumn{2}{|c|}{ SN.PP } & 9.19 & 3.97 & 9.04 & 4.14 & -0.15 & 1.60 \\
\hline \multicolumn{2}{|c|}{ ENA-SN } & 54.77 & 3.76 & 56.50 & 4.37 & 1.73 & 1.48 \\
\hline \multicolumn{2}{|c|}{ ENP-SN } & 46.15 & 3.21 & 47.58 & 4.19 & 1.42 & 1.78 \\
\hline \multicolumn{2}{|c|}{ Nperp-A } & -1.04 & 3.61 & -0.35 & 3.23 & 0.69 & 2.10 \\
\hline \multicolumn{2}{|c|}{ Co-A } & 89.88 & 4.75 & 92.38 & 5.52 & 2.50 & 2.19 \\
\hline
\end{tabular}

Tabela 10 - Resultado dos contrastes para a variável SNA.

\begin{tabular}{|c|c|c|c|c|c|}
\hline Variável & Contraste & $\begin{array}{l}\text { Fonte de } \\
\text { variabi- } \\
\text { lidade }\end{array}$ & $\begin{array}{l}\text { Quadra- } \\
\text { do } \\
\text { Médio }\end{array}$ & $\mathbf{F}$ & $\begin{array}{c}\text { Nível de } \\
\text { significância } \\
\text { (p) }\end{array}$ \\
\hline SNA & 1 e 2 & T $1-\mathrm{T} 2$ & 8.1737 & 17.87 & $0.0002 * *$ \\
\hline SNA & 1 e 2 & $\begin{array}{c}\text { Grupo x } \\
\text { Tempo }\end{array}$ & 0.9157 & 2.00 & 0.1677 NS \\
\hline SNA & 2 e 3 & T $2-\mathrm{T} 3$ & 1.4062 & 0.83 & $0.3606 \mathrm{NS}$ \\
\hline SNA & 2 e 3 & $\begin{array}{c}\text { Grupo x } \\
\text { Tempo }\end{array}$ & 9.3553 & 5.61 & $0.0248 *$ \\
\hline SNA & 3 e 1 & T $3-\mathrm{T} 1$ & 2.7993 & 1.22 & 0.2779 NS \\
\hline SNA & 3 e 1 & $\begin{array}{l}\text { Grupo } \mathrm{x} \\
\text { Tempo }\end{array}$ & 4.5412 & 1.98 & 0.1696 NS \\
\hline
\end{tabular}

NS: não significante, ${ }^{*}$ : significante ao $5 \%(p<0.05),{ }^{* *}$ : significante ao $1 \%(p<0.01)$

Tabela 12 - Análise de variância de medidas repetidas para a variável ENA-SN.

\begin{tabular}{ccccc}
\hline Variável & $\begin{array}{c}\text { Fonte de varia- } \\
\text { bilidade }\end{array}$ & $\begin{array}{c}\text { Quadrado } \\
\text { Médio }\end{array}$ & $\mathbf{F}$ & $\begin{array}{c}\text { Nível de signifi- } \\
\text { cância (p) }\end{array}$ \\
\hline ENA-SN & Grupo & 1.5389 & 0.05 & $0.8225 \mathrm{NS}$ \\
ENA-SN & Tempo & 27.9662 & 22.84 & 0.0001 ** \\
ENA-SN & Grupo x Tempo & 0.3856 & 0.31 & $0.6813 \mathrm{NS}$ \\
\hline
\end{tabular}

NS: não significante, ${ }^{*}$ : significante ao $5 \%(p<0.05),{ }^{* *}$ : significante ao $1 \%(p<0.01)$ 
Tabela 13 - Resultado dos contrastes para a variável ENASN.

\begin{tabular}{|c|c|c|c|c|c|}
\hline Variável & Contraste & $\begin{array}{c}\text { Fonte de } \\
\text { variabili- } \\
\text { dade }\end{array}$ & $\begin{array}{c}\text { Quadra- } \\
\text { do } \\
\text { Médio }\end{array}$ & $\mathbf{F}$ & $\begin{array}{c}\text { Nível de } \\
\text { sig- } \\
\text { nificân- } \\
\text { cia (p) }\end{array}$ \\
\hline ENA-SN & 1 e 2 & $\mathrm{~T} 1-\mathrm{T} 2$ & 34.3260 & 31.74 & $0.0001^{* *}$ \\
\hline ENA-SN & 1 e 2 & $\begin{array}{c}\text { Grupo x } \\
\text { Tempo }\end{array}$ & 0.0034 & 0.00 & $\begin{array}{c}0.9554 \\
\text { NS }\end{array}$ \\
\hline ENA-SN & 2 e 3 & Т $2-$ T 3 & 22.0568 & 8.19 & $0.0078 * *$ \\
\hline ENA-SN & 2 e 3 & $\begin{array}{c}\text { Grupo x } \\
\text { Tempo }\end{array}$ & 1.2181 & 0.45 & $\begin{array}{c}0.5067 \\
\text { NS }\end{array}$ \\
\hline ENA-SN & 1 e 3 & T $1-\mathrm{T} 3$ & 111.4145 & 31.19 & $0.0001^{* *}$ \\
\hline ENA-SN & 1 e 3 & $\begin{array}{c}\text { Grupo x } \\
\text { Tempo }\end{array}$ & 1.0919 & 0.31 & $\begin{array}{c}0.5846 \\
\text { NS }\end{array}$ \\
\hline
\end{tabular}

NS: não significante, ${ }^{*}$ : significante ao $5 \%(p<0.05),{ }^{* *}$ : significante ao $1 \%(p<0.01)$

Tabela 14 - Análise de variância de medidas repetidas para a variável ENP-SN.

\begin{tabular}{ccccc}
\hline Variável & $\begin{array}{c}\text { Fonte de } \\
\text { variabili- } \\
\text { dade }\end{array}$ & $\begin{array}{c}\text { Quadrado } \\
\text { Médio }\end{array}$ & F & $\begin{array}{c}\text { Nível de sig- } \\
\text { nificância (p) }\end{array}$ \\
\hline ENP-SN & Grupo & 9.5969 & 0.31 & $0.5793 \mathrm{NS}$ \\
ENP-SN & Tempo & 23.6792 & 21.99 & $0.0001 * *$ \\
ENP-SN & $\begin{array}{c}\text { Grupo } \mathrm{x} \\
\text { Tempo }\end{array}$ & 0.9964 & 0.93 & $0.3992 \mathrm{NS}$ \\
\hline
\end{tabular}

NS: não significante, ${ }^{*}$ : significante ao $5 \%(p<0.05),{ }^{* *}$ : significante ao $1 \%(p<0.01)$

Tabela 15 - Resultado dos contrastes para a variável ENP-SN.

\begin{tabular}{|c|c|c|c|c|c|}
\hline Variável & $\begin{array}{l}\text { Con- } \\
\text { traste }\end{array}$ & $\begin{array}{c}\text { Fonte de } \\
\text { variabili- } \\
\text { dade }\end{array}$ & $\begin{array}{l}\text { Quadra- } \\
\text { do Médio }\end{array}$ & $\mathbf{F}$ & $\begin{array}{l}\text { Nível de } \\
\text { significân- } \\
\text { cia (p) }\end{array}$ \\
\hline ENP-SN & 1 e 2 & $\mathrm{~T} 1-\mathrm{T} 2$ & 40.5716 & 26.78 & $0.0001 * *$ \\
\hline ENP-SN & 1 e 2 & $\begin{array}{c}\text { Grupo x } \\
\text { Tempo }\end{array}$ & 2.9587 & 1.95 & 0.1728 NS \\
\hline ENP-SN & 2 e 3 & $\mathrm{~T} 2-\mathrm{T} 3$ & 10.1616 & 4.95 & 0.0341 * \\
\hline ENP-SN & 2 e 3 & $\begin{array}{l}\text { Grupo x } \\
\text { Tempo }\end{array}$ & 0.0003 & 0.00 & 0.9903 NS \\
\hline ENP-SN & 1 e 3 & T 1 - T 3 & 91.3422 & 31.58 & $0.0001 * *$ \\
\hline ENP-SN & 1 e 3 & $\begin{array}{l}\text { Grupo x } \\
\text { Tempo }\end{array}$ & 3.0196 & 1.04 & $0.3153 \mathrm{NS}$ \\
\hline
\end{tabular}

NS: não significante, ${ }^{*}$ : significante ao $5 \%(p<0.05),{ }^{* *}$ : significante ao $1 \%(p<0.01)$
Tabela 16 - Análise de variância de medidas repetidas para a variável Nperp-A.

\begin{tabular}{ccccc}
\hline Variável & $\begin{array}{c}\text { Fonte de varia- } \\
\text { bilidade }\end{array}$ & $\begin{array}{c}\text { Quadrado } \\
\text { Médio }\end{array}$ & F & $\begin{array}{c}\text { Nível de sig- } \\
\text { nificância (p) }\end{array}$ \\
\hline Nperp-A & Grupo & 87.3835 & 2.19 & $0.1493 \mathrm{NS}$ \\
Nperp-A & Tempo & 7.1952 & 2.20 & $0.1196 \mathrm{NS}$ \\
Nperp-A & Grupo x Tempo & 1.0017 & 0.31 & $0.7370 \mathrm{NS}$ \\
\hline
\end{tabular}

NS: não significante, ${ }^{*}$ : significante ao $5 \%(p<0.05),{ }^{* *}$ : significante ao $1 \%(p<0.01)$

Tabela 17 - Análise de variância de medidas repetidas para a variável $\mathrm{Co}-\mathrm{A}$.

\begin{tabular}{ccccc}
\hline Variável & $\begin{array}{c}\text { Fonte de } \\
\text { variabili- } \\
\text { dade }\end{array}$ & $\begin{array}{c}\text { Quadrado } \\
\text { Médio }\end{array}$ & F & $\begin{array}{c}\text { Nível de sig- } \\
\text { nificância (p) }\end{array}$ \\
\hline Co-A & Grupo & 26.9976 & 0.43 & $0.5179 \mathrm{NS}$ \\
Co-A & Tempo & 37.3173 & 19.73 & $0.0001^{* *}$ \\
Co-A & $\begin{array}{c}\text { Grupo } x \\
\text { Tempo }\end{array}$ & 0.8012 & 0.42 & $0.6381 \mathrm{NS}$ \\
\hline
\end{tabular}

NS: não significante, ${ }^{*}$ : significante ao $5 \%(p<0.05),{ }^{* *}$ : significante ao $1 \%(p<0.01)$

Tabela 18 - Resultado dos contrastes para a variável Co-A.

\begin{tabular}{|c|c|c|c|c|c|}
\hline Variável & Contraste & $\begin{array}{c}\text { Fonte de } \\
\text { variabili- } \\
\text { dade }\end{array}$ & $\begin{array}{l}\text { Quadra- } \\
\text { do Médio }\end{array}$ & $\mathbf{F}$ & $\begin{array}{c}\text { Nível de } \\
\text { significân- } \\
\text { cia (p) }\end{array}$ \\
\hline Co-A & 1 e 2 & $\mathrm{~T} 1-\mathrm{T} 2$ & 33.0990 & 15.69 & $0.0004^{* *}$ \\
\hline Co-A & 1 e 2 & $\begin{array}{c}\text { Grupo x } \\
\text { Tempo }\end{array}$ & 0.0022 & 0.00 & 0.9744 NS \\
\hline Co-A & 2 e 3 & T $2-\mathrm{T} 3$ & 41.7011 & 9.30 & $0.0049 * *$ \\
\hline Co-A & 2 e 3 & $\begin{array}{l}\text { Grupo x } \\
\text { Tempo }\end{array}$ & 2.4753 & 0.55 & $0.4634 \mathrm{NS}$ \\
\hline Co-A & 1 e 3 & T $1-\mathrm{T} 3$ & 149.1039 & 31.35 & $0.0001 * *$ \\
\hline Co-A & 1 e 3 & $\begin{array}{l}\text { Grupo x } \\
\text { Tempo }\end{array}$ & 2.3297 & 0.49 & $0.4896 \mathrm{NS}$ \\
\hline
\end{tabular}

NS: não significante, ${ }^{*}$ : significante ao $5 \%(p<0.05),{ }^{* *}$ : significante ao $1 \%(\mathrm{p}<0.01)$ 
inicialmente, na Universidade de Illinois, inaugurando uma nova era na Ortodontia americana. Os clássicos trabalhos publicados por Haas ${ }^{17,18,20}$, a partir da década de 60, alcançaram grande repercussão e foram capazes de ressuscitar o invento de Angell ${ }^{2}$, demonstrando cientificamente sua potencialidade terapêutica mediante estudos histológicos em animais e avaliações cefalométricas e clínicas em animais e seres humanos, padronizando as condutas adotadas atualmente.

A deficiência transversal da largura maxilar pode ser oriunda de fatores genéticos ou ambientais ${ }^{6}$, envolvendo apenas os segmentos dentários posteriores, com uma grande inclinação para o lado palatino, ou estar associada a um comprometimento esquelético da maxila, apresentando um aspecto atrésico, com uma abóboda palatina ogival e estreita ${ }^{15}$, necessitando, para sua correção, de uma expansão capaz de promover uma alteração ortopédica dos segmentos maxilares, mantendo a integridade dos tecidos envolvidos e minimizando os efeitos de inclinação dentária ${ }^{6,19,36}$.

A disjunção rápida da sutura palatina mediana preenche estes preceitos, restabelecendo as dimensões transversais da maxila e seu correspondente arco dentário ${ }^{37}$, mediante a abertura da sutura palatina mediana associada às reações ortopédicas em outras suturas faciais e a uma pequena movimentação dos dentes póstero-superiores ${ }^{36,37}$.

Desde os primeiros relatos sobre a disjunção da sutura palatina mediana ${ }^{2}$ até o momento atual, vários estudiosos como Babcock ${ }^{4}$; Biederman $^{5}$; Christie, Ruedemann ${ }^{12}$; Cohen, Silverman ${ }^{13}$; Haas $^{18}$; Hilgers ${ }^{23}$; Howe ${ }^{24}$; Moss ${ }^{30,31}$; Sarver, Johnston $^{34}$ e Timms ${ }^{38}$ propuseram diversos tipos de aparelhos disjuntores rápidos da maxila. Apesar das diferenças e das modificações impostas nos diversos aparelhos, o princípio mecânico básico não se alterou, culminando nos resultados finais das disjunções palatinas.

Assim sendo, com o intuito de se avaliar cefalometricamente o posicionamento maxilar após os efeitos da disjunção rápida da sutura palatina mediana e a efetividade dos aparelhos disjuntores tipo Haas e tipo Hyrax, propusemo-nos a realizar este estudo.

Em razão dos inúmeros dados fornecidos e da complexidade em se avaliar todas as variáveis dos dois grupos, simultaneamente, nos três momentos do tratamento em estudo, procurou-se, para melhor entendimento e compreensão do leitor, analisar cada variável individualmente e, na medida do possível, no total do complexo craniofacial.

\section{Variável SNA}

O comportamento da maxila no sentido ântero-posterior foi averiguado em relação à base do crânio, em ambos os grupos, pela medida angular SNA. As tabelas 1 e 2 demonstram respectivamente, para o Grupo I (Haas) e Grupo II (Hyrax), um aumento pouco expressivo no valor médio de SNA na fase pós-disjunção, sendo que, na fase final de nivelamento dos arcos dentários esse valor retrocedeu no Grupo I e aumentou discretamente no Grupo II. A Análise de Variância mostrou valores próximos dos significantes na interação Grupo $x$ Tempo ( $\mathrm{p}=0.0604)$, ou seja, o comportamento de SNA ao longo do tempo poderia ser considerado diferente para os dois grupos da amostra, portanto, foram calculados contrastes para cada grupo e não se verificaram diferenças estatisticamente significantes entre eles. Os contrastes para cada tempo resultaram em uma diferença significante entre as fases início de tratamento e pós-disjunção, para ambos os grupos, entretanto, não se verificou significado estatístico na diferença entre o início de tratamento e o final do nivelamento (Tab. 9, 10; Gráf. 1).

Os resultados cefalométricos e estatísticos obtidos nesse estudo apresentaram concordância com os autores Bozola, Scanavini ${ }^{7}$; Byrum Jr. ${ }^{9}$; Davis, Kronman ${ }^{14}$; Haas ${ }^{17,18,19,20,21}$; Marchi2 ${ }^{28}$; Silva Filho et al. $^{36}$; Wertz e Dreskin ${ }^{41}$ que relatam o deslocamento anterior desta base óssea, após os efeitos da disjunção rápida da sutura palatina mediana. Verificou-se, entretanto, que os valores de SNA 


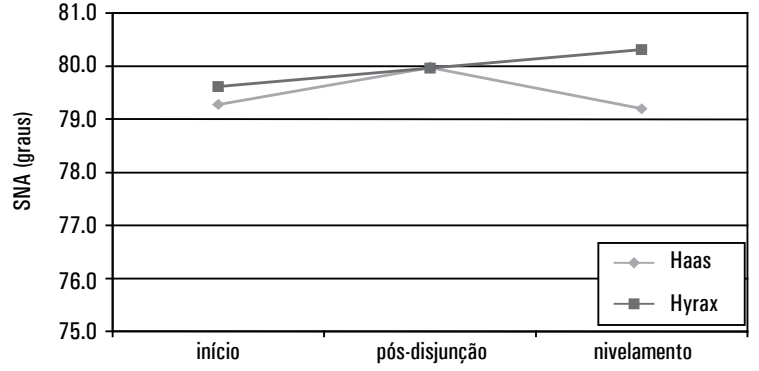

GRÁFICO 1 - Alterações da variável SNA, durante as fases estudadas, nos Grupos I (Haas) e II (Hyrax).

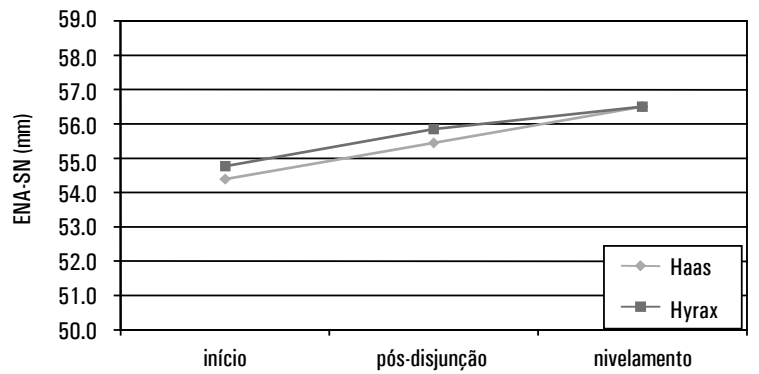

GRÁFICO 3 - Alterações da variável ENA-SN, durante as fases estudadas, nos Grupos I (Haas) e II (Hyrax).

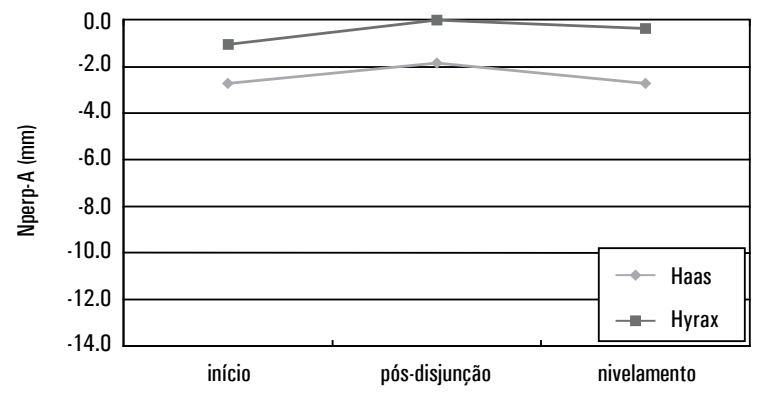

GRÁFICO 5 - Alterações da variável Nperp-A, durante as fases estudadas, nos Grupos I (Haas) e II (Hyrax).

obtidos para o final de nivelamento, em ambos os grupos, eram semelhantes estatisticamente aos valores pré-disjunção.

\section{Variável SN.PP}

As alterações verticais no posicionamento da maxila em relação à base do crânio, decorrentes do

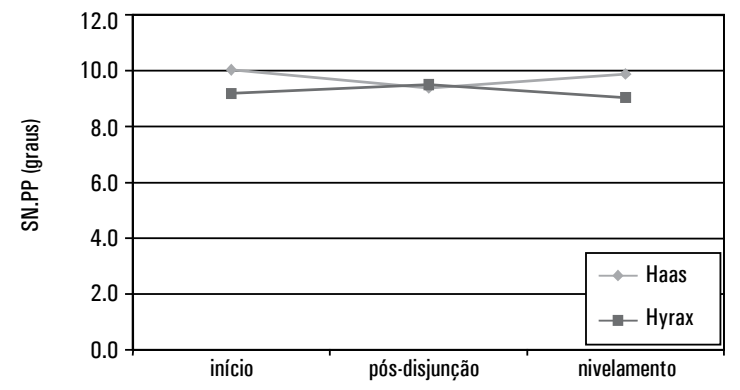

GRÁFICO 2 - Alterações da variável SN.PP, durante as fases estudadas, nos Grupos I (Haas) e II (Hyrax).

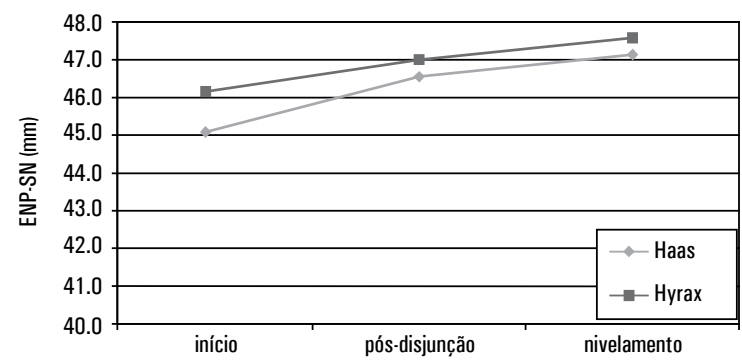

GRÁFICO 4 - Alterações da variável ENP-SN, durante as fases estudadas, nos Grupos I (Haas) e II (Hyrax).

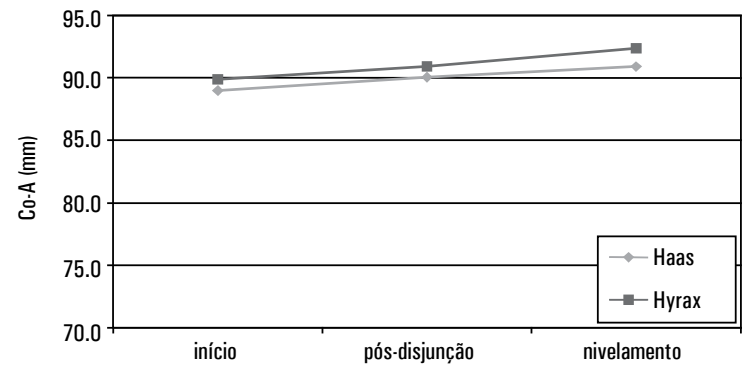

GRÁFICO 6 - Alterações da variável Co-A, durante as fases estudadas, nos Grupos I (Haas) e II (Hyrax).

procedimento de disjunção rápida da sutura palatina mediana, foram verificadas pela medida angular SN.PP. As tabelas 1 e 2 apresentam os valores médios de SN.PP durante as fases do tratamento em estudo, para os Grupos I e II respectivamente. Observou-se uma pequena diminuição no valor médio desta variável na fase pós-disjunção para 
o Grupo I (Haas) e um discreto aumento para o Grupo II (Hyrax), sendo que, na fase final de nivelamento, verificou-se a normalização desses valores em ambos os grupos.

Estatisticamente, a Análise de Variância para SN.PP não apresentou significância na interação Grupo x Tempo, efeito entre grupos ou efeito entre tempos, portanto, o plano palatino não sofreu rotação estatisticamente significante, em relação à linha S-N, em nenhum momento do tratamento, tanto para o Grupo I quanto para o Grupo II (Tab. 11, Gráf. 2), verificado também por Silva Filho et al. ${ }^{35}$, mas não apresentando concordância com outros estudos como os de Davis, Kronman ${ }^{14}$; Silva Filho et al. ${ }^{37}$ e White ${ }^{42}$.

\section{Variável ENA-SN}

As alterações no posicionamento vertical da maxila em relação à base do crânio foram verificadas pela distância linear ENA-SN, pela projeção ortogonal do ponto cefalométrico ENA (espinha nasal anterior) a linha S-N (base do crânio). As tabelas 1 e 2 apresentam dados que demonstram um aumento gradativo da distância linear ENA-SN, tanto para o Grupo I (Haas) quanto para o Grupo II (Hyrax), durante as fases estudadas, resultando em um deslocamento para baixo da maxila, corroborando com a literatura ${ }^{7,9,14,17,18,19,20,22,26,28,31,34,35,37,40,41}$.

A Análise de Variância mostrou-se significante no efeito entre tempos, deste modo, através dos contrastes, verificou-se que o aumento do valor médio de ENA-SN entre as fases início de tratamento e pós-disjunção, bem como o aumento desse valor entre as fases pós-disjunção e final de nivelamento, início de tratamento e final do nivelamento, foi estatisticamente significante para os dois grupos da amostra (Tab. 12, 13; Gráf. 3).

\section{Variável ENP-SN}

A distância linear ENP-SN, pela projeção ortogonal do ponto cefalométrico ENP (espinha nasal posterior) a linha S-N (base do crânio), auxiliou na verificação das alterações no posicionamen- to vertical da maxila durante as fases estudadas. As tabelas 1 e 2 apresentam dados que demonstram alterações semelhantes àquelas descritas para a variável ENA-SN, isto é, em ambos os grupos foi observado um aumento gradativo dos valores médios de ENP-SN, nas fases de pós-disjunção e final de nivelamento, o que resultou em um deslocamento para baixo da maxila, que acompanhou a disposição das suturas maxilofaciais, que sofreram uma desestruturação com a disjunção rápida $\mathrm{da}$ sutura palatina mediana ${ }^{6,40}$.

A diferença estatisticamente significante entre tempos determinou a realização de contrastes para verificar entre quais tempos existiram diferenças, constatou-se que o aumento dos valores médios de ENP-SN, entre as fases início de tratamento e pós-disjunção, bem como, entre as fases pós-disjunção e final de nivelamento e, início de tratamento e final de nivelamento, apresentaram significado estatístico para o Grupo I e Grupo II (Tab. 14, 15; Gráf. 4).

\section{Variável Nperp-A}

O posicionamento da maxila no sentido ânteroposterior foi também verificado pela grandeza cefalométrica Nperp-A. Os dados apresentados nas tabelas 1 e 2 demonstram alterações semelhantes para os Grupos I e II, no posicionamento da maxila, denotando que o ponto A, após movimentar-se para frente durante a fase ativa da disjunção da sutura palatina mediana, retomou valores próximos aos iniciais na fase final de nivelamento. Os resultados apresentam concordância com diversos trabalhos citados anteriormente $7,9,14,17,18,19,20,21,28,30,36,41$ mas não corroboraram com os obtidos por Silva Filho et al..$^{35}$, que também utilizaram uma amostra de pacientes que apresentavam dentição permanente, em que não se constatou a movimentação para anterior da maxila com resultados estatisticamente significantes, após o procedimento da disjunção rápida.

A Análise de Variância não mostrou significado estatístico para os efeitos de interação Grupo x Tempo, 
efeito de comparação entre grupos ou comparação entre tempos, portanto, não foram realizados contrastes (Tab. 16, Gráf. 5).

\section{Variável Co-A}

O comprimento efetivo da maxila, mensurado pela medida linear Co-A, adotada por McNamara $\mathrm{Jr}^{27}$, apresentou um aumento gradativo nos Grupos I e II, durante as fases estudadas, conforme os dados apresentados nas tabelas 1 e 2 . Teoricamente, o aumento médio desta medida em $1.06 \mathrm{~mm}$ para o Grupo I (Haas) (Tab. 3) e $1.04 \mathrm{~mm}$ para o Grupo II (Hyrax) (Tab. 6), em apenas 11 a 14 dias de disjunção ativa, não foi devido às alterações impostas pelo crescimento craniofacial, visto que o prazo para esta ocorrência foi muito curto.

A análise estatística revelou que a variável Co-A apresentou alterações semelhantes ao longo do tempo para os dois grupos da amostra, de modo que a diferença entre as fases início de tratamento e pós-disjunção, bem como entre as fases pósdisjunção e final de nivelamento, e ainda entre as fases início de tratamento e final de nivelamento, apresentaram-se estatisticamente significantes (Tab. 17, 18; Gráf. 6). Pressupõe-se, por esta variável cefalométrica, que houve uma pequena movimentação do ponto A para frente, durante as fases estudadas, embora outras medidas, já comentadas anteriormente, como SNA e Nperp-A, não apresentassem este deslocamento para anterior, com um resultado estatisticamente significativo, entre as fases início do tratamento e final do nivelamento.

\section{CONSIDERAÇÕES FINAIS}

Os efeitos ortopédicos da disjunção rápida da sutura palatina mediana são bem conhecidos e descritos na literatura $7,16,17,18,19,20,21,36,40$. À medida que o parafuso expansor é ativado, ocorre a disjunção da sutura palatina mediana, ocasionando um aumento real do perímetro do arco dentário superior ${ }^{1}$ acompanhado de boa estabilidade ${ }^{21}$. Essas respostas ortopédicas podem ser comprovadas nos planos horizontal e frontal ${ }^{6,18,19,21,34,36,37,40}$.
No plano horizontal, a abertura da sutura palatina mediana assume uma forma triangular ${ }^{6,40,42}$, visualizada por meio de radiografias oclusais, com o vértice localizado na união das lâminas horizontais dos ossos palatinos e a base voltada para a região entre os incisivos centrais ${ }^{6,40}$. Associada a essa abertura da sutura, ocorre uma inclinação vestibular dos processos alveolares, $6,18,19,21,40,41$ e dos dentes póstero-superiores ${ }^{1,3,6,7,17,18,21,26,40,41}$. No plano frontal, a separação dos maxilares obedece à mesma conformação geométrica, com o ponto de fulcro localizado próximo à sutura frontonasal ${ }^{22,36,40,41}$, apresentando uma maior quantidade de expansão ao nível do plano oclusal, diminuindo em direção aos processos alveolares, osso basal da maxila e cavidade nasal ${ }^{6,18,34,36,37,40}$.

Pela avaliação estatística do conjunto de variáveis empregadas neste estudo, de uma maneira geral, não se observaram diferenças entre os resultados obtidos para os dois tipos de aparelhos empregados que apresentassem influência direta sobre os procedimentos clínicos. Os dois tipos de aparelhos disjuntores demonstraram a sua capacidade em promover a disjunção rápida da sutura palatina mediana.

As variáveis avaliadas nesse estudo permitiram acompanhar o comportamento sagital e vertical da maxila frente à expansão rápida de sua sutura palatina.

As modificações verticais induzidas na maxila, decorrentes da disjunção rápida da sutura palatina mediana, verificadas pelas medidas ENA-SN e ENP-SN, comprovaram o seu deslocamento inferior, ratificando o aumento da altura facial anterior e posterior, sem a ocorrência de rotação do plano palatino, visto que os valores obtidos para a variável SN.PP não apresentaram significado estatístico, em nenhuma fase do tratamento em estudo.

No sentido sagital, os valores obtidos para SNA e Nperp-A demonstraram um deslocamento anterior da maxila em relação à base do crânio logo após a disjunção. No entanto, esse movimento não apresentou estabilidade e os 
valores dessas variáveis retornaram aos obtidos na fase de pré-disjunção. A variável CoA apresentou comportamento diferente e manteve a tendência de aumento, verificada na fase de pós-disjunção, também ao final do nivelamento. Deve-se considerar que as duas primeiras variáveis avaliam a localização ântero-posterior da maxila em relação à base do crânio, não sendo influenciadas pela sua posição vertical, enquanto a terceira avalia o comprimento efetivo da maxila, medido obliquamente entre os pontos condílio e subespinhal, distância essa influenciada pela posição vertical da maxila. Pode-se então explicar a diferença de comportamento dessas variáveis, pois a maxila não alterou a sua posição sagital entre as fases de pré-disjunção e final de nivelamento, entretanto apresentou deslocamento inferior, demonstrado pelo incremento dos valores de ENA-SN e ENP$\mathrm{SN}$, que aumentou a distância entre os pontos $\mathrm{Co}$ e A. Conclui-se, portanto, que as variáveis SNA e Nperp-A são mais adequadas para avaliação da posição sagital da maxila, pois são menos infuenciadas pela alteração vertical.

\section{CONCLUSÕES}

Segundo a metodologia empregada e diante da análise e discussão dos resultados obtidos, julgamos válido concluir que:

1) o posicionamento da maxila, no sentido ântero-posterior em relação à base do crânio, sofreu modificações semelhantes para ambos os grupos, com a ocorrência de avanço maxilar no fase pósdisjunção, evidenciado pelo comportamento estatístico das variáveis SNA e Nperp-A, seguida de um retorno aos valores iniciais na fase final de nivelamento. A variável CoA manteve, ao final do nivelamento, o incremento verificado pós-disjunção, devido à influência do aumento vertical sobre a medição dessa variável.

2) O posicionamento da maxila no sentido vertical também apresentou modificações semelhantes para ambos os grupos, com a ocorrência de deslocamento vertical da maxila para baixo, sem rotação, evidenciado pelo comportamento estatístico das variáveis ENA-SN, ENP-SN e SN.PP.

Enviado em: Setembro de 2003 Revisado e aceito: Janeiro de 2004

\title{
Comparative evaluation of maxilar effects of rapid maxilar expansion with Haas and Hyrax appliances
}

\begin{abstract}
Aim: the purpose of this cephalometric study was to evaluate, by lateral cephalograms, the changes in maxilar positioning after rapid disjunction of the midpalatal suture, following the use of two types of maxillary disjunction appliances, checked in different phases, and the likely differences between the two appliances Haas and Hyrax. Methods: the sample comprised of 93 lateral cephalograms, taken before treatment (pre-disjunction), immediately after disjunction and at the end of levelling, obtained from 31 brazilian youths with both genres and average age of 13 years and 2 months. Results and Conclusions: both types of appliances showed similar results, with anterior and lower displacement of maxila right after disjunction. Lower displacement was without rotation, and mainttened stable until the end of levelling. Anterior displacement, however, was not stable and cephalometric measurements like SNA and Nperp-A tended to returned to initial values at the end of levelling.

Key words: Rapid maxillary expansion. Cephalometry. Maxilla.

1. ADKINS, M. D.; NANDA, R.S.; CURRIER, G. F. Arch perimeter changes on rapid palatal expansion. Am J Orthod Dentofacial Orthop, St. Louis, v. 97, no. 3, p. 194-199, Mar.1990.

2. ANGELL, E. H. Treatment of irregularity of the permanent or adult teeth. Dent Cosmos, Philadelphia, v. 1, p. 540-544, 599-601, 1860.

3. ASANZA, S.; CISNEROS, G. J.; NIEBERG, L. G. Comparison of Hyrax and bonded expansion appliances. Angle Orthod, Appleton, v. 67, no. 1, p. 15-22, 1997.

4. BABCOCK, S. H. The screw expansion plate. Dent Rec, London,
\end{abstract}

\section{REFERÊNCIAS}


v. 31, p. 588-590, 596-599, 1911.

5. BIEDERMAN, W. A hygienic appliance for rapid expansion. J Pract Orthod, Hempstead, v. 2, no. 2, p. 67-70, Feb.1968.

6. BISHARA, S. E.; STANLEY, R. N. Maxillary expansion: clinical implications. Am J Orthod Dentofacial Orthop, St. Louis, v. 91, no. 1, p. 3-14, Jan. 1987.

7. BOZOLA, B. C.; SCANAVINI, M. A. Disjunção rápida da sutura palatina mediana e sua influência sobre os ossos da face. Rev Ortodontia Paranaense, Curitiba, v. 7, n. 1, p. 29-41, jan./jun. 1986.

8. BROWN, G. V. I. The application of orthodontic principles to the prevention of nasal disease. Dent Cosmos, Philadelphia, v. 45, no.10, p. 765-775, Oct. 1903.

9. BYRUM JR., A. G. Evaluation of anterior-posterior and vertical skeletal change versus dental change in rapid palatal expansion cases as studied by lateral cephalograms. Am J Orthod, St. Louis, v. 60, no. 4, p. 419, Oct. 1971.

10. CAMERON C. G.; FRANCHI, L.; BACCETTI, T.; McNAMARA Jr. J. A. Long term effects of rapid maxillary expansion: a posteroanterior cephalometric evaluation. Am J Orthod Dentofacial Orthop, St. Louis, v. 121, no. 2, p.129-135, Feb. 2002

11. CAPELOZZA FILHO, L. et al. Expansão rápida da maxila cirurgicamente assistida. Ortodontia, São Paulo, v. 27, n. 1, p. 21-30, jan./abr. 1994

12. CHRISTIE, T. E.; RUEDEMANN, P. P. Rapid separation of the midpalatal suture. J Pract Orthod, Hempstead, v. 1, no. 1, p.19-21, Sept.1967.

13. COHEN, M.; SILVERMAN, E. A new and simple palate splitting device. J Clin Orthod, Hempstead, v. 7, no. 6, p. 368-369, June 1973.

14. DAVIS, W. M.; KRONMAN, J. H. Anatomical changes induced by splitting of the midpalatal suture. Angle Orthod, Appleton, v. 39, no. 2, p. 126-132, Apr. 1969.

15. DIPAOLO, R. J. Thoughts on palatal expansion. J Clin Orthod, Hempstead, v. 4, no. 9, p. 493-497, Sept. 1970.

16. GARDNER, G. E.; KRONMAN, J. H. Cranioskeletal displacements caused by rapid palatal expansion in the Rhesus Monkey. Am J Orthod, St. Louis, v. 59, no. 2, p. 146-155, Feb. 1971.

17. HAAS, A. J. Rapid expansion of the maxillary dental arch and nasal cavity by opening the midpalatal suture. Angle Orthod, Appleton, v. 31, no. 2, p. 73-90, Apr. 1961.

18. HAAS, A. J. The treatment of maxillary deficiency by opening the midpalatal suture. Angle Orthod, Appleton, v. 35, no. 3, p. 200-217, July 1965.

19. HAAS, A. J. Palatal expansion: just the beginning of dentofacial orthopedics. Am J Orthod, St. Louis, v. 57, no. 3, p. 219-255, Mar. 1970

20. HAAS, A. J. Interviews. J Clin Orthod, Hempstead, v. 7, no. 4, p. 227-245, Apr. 1973

21. HAAS, A. J. Long-term post-treatment evaluation of rapid palatal expansion. Angle Orthod, Appleton, v. 50, no. 3, p.189-217, July 1980 .

22. HEFLIN, B. M. A three-dimensional cephalometric study of the influence of expansion of the midpalatal suture on the bones of the face. Am J Orthod, St. Louis, v. 57, no. 2, p. 194-195, Feb. 1970.

23. HILGERS, J. J. A palatal expansion appliance for non-compliance therapy. J Clin Orthod, Hempstead, v. 25, no. 8, p. 491-497, Aug. 1991

24. HOWE, R. P. Palatal expansion using a bonded appliance. Am J Orthod, St. Louis, v. 82, no. 6, p. 464-468, Dec.1982.

25. LÉON, A. P. F. et al. Aparelho expansor colado com cobertura acrílica para o controle vertical, durante a expansão rápida da maxila: apresentação de um caso clínico. R Dental Press Ortodon Ortop Maxilar, Maringá, v. 3, n. 3, p. 25-33, maio/jun. 1998.

26. LINDER-ARONSON, S.; LINDGREN, J. The skeletal and dental effects of rapid maxillary expansion. Br J Orthod, Oxford, v. 6, no. 1, p. 25-29, Jan. 1979

27. McNAMARA JR., J. A. A method of cephalometric evaluation. Am J Orthod, St. Louis, v. 86, no. 6, p. 449-469, Dec.1984.
28. MARCHI L. C. Estudo cefalométrico-radiográfico das modificações no padrão dentofacial em norma lateral e frontal e de medidas transversais, em modelos, decorrentes da disjunção rápida da sutura palatina mediana. 1988. 98 f. Dissertação (Mestrado)- Faculdade de Odontologia, Universidade Metodista de São Paulo, Piracicaba, 1988.

29. MAZZIERO, E. T. Estudo cefalométrico, em norma frontal, das alterações dentoesqueléticas após a expansão rápida da maxila, em pacientes na faixa etária de $\mathbf{1 0}$ a $\mathbf{1 6}$ anos e $\mathbf{2}$ meses. 1994. 128 f. Dissertação (Mestrado)-Faculdade de Odontologia de Bauru, Universidade de São Paulo, Bauru, 1994.

30. MOSS, J. P. Rapid expansion of the maxillary arch. Part I. J Pract Orthod, Hempstead, v. 2, no. 4, p.165-171, Apr. 1968.

31. MOSS, J. P. Rapid expansion of the maxillary arch. Part II. J Pract Orthod, Hempstead, v. 2, no. 5, p. 215-223, May 1968.

32. PFAFF, W. Stenosis of the nasal cavity caused by contraction of the palate and abnormal position of the teeth: treatment by expansion of the maxilla. Dent Cosmos, Philadelphia, v. 47, no. 6, p. 570-573, June 1905.

33. REED, N.; GHOSH, J.; NANDA, R. S. Comparison of treatment outcomes with banded and bonded RPE appliances. Am J Orthod Dentofacial Orthop, St. Louis, v. 116, no.1, p. 31-40, July 1999.

34. SARVER, D. M.; JOHNSTON, M. W. Skeletal changes in vertical and anterior displacement of the maxilla with bonded rapid palatal expansion appliances. Am J Orthod Dentofacial Orthop, St. Louis, v. 95, no. 6, p.462-466, June 1989

35. SILVA FILHO, O. G. et al. Expansão rápida da maxila na dentadura permanente: avaliação cefalométrica. Ortodontia, São Paulo, v. 27, n. 2, p. 68-76, maio/ago.1994.

36. SILVA FILHO, O. G.; VALLADARES NETO, J.; ALMEIDA, R. R. Early correction of posterior crossbite: biomechanical characteristics of the appliances. J Pedod, Birmingham, v. 13, no. 3, p.195-221, Spring 1989.

37. SILVA FILHO, O. G.; VILLAS BOAS, M. C.; CAPELOZZA FILHO, L. Rapid maxillary expansion in the primary and mixed dentitions: a cephalometric evaluation. Am J Orthod Dentofacial Orthop, St. Louis, v. 100, no. 2, p.171-179, Aug.1991.

38. TIMMS, D. J. Rapid maxillary expansion. Chicago: Quintessence, 1981. p. 16-37

39. VELAZQUEZ, P.; BENITO, E.; BRAVO, L. A. Rapid maxillary expansion: a study of the long-term effects. Am J Orthod Dentofacial Orthop, St. Louis, v. 109, no. 4, p. 361-367, Apr. 1996.

40. WERTZ, R. A. Skeletal and dental changes accompanying rapid midpalatal suture opening. Am J Orthod, St. Louis, v. 58, no. 1, p. 41-66, July 1970 .

41. WERTZ, R. A.; DRESKIN, M. Midpalatal suture opening: a normative study. Am J Orthod, St. Louis, v. 71, no. 4, p. 367-381, Apr. 1977.

42. WHITE, R. E. A cephalometric appraisal of changes in the maxillofacial complex resulting from palatal suture expansion utilizing fixed appliance therapy. Am J Orthod, St. Louis, v. 61, no. 5, p. 527-528, May 1972. Abstract.

Endereço para correspondência

Sílvia Augusta Braga Reis

Rua Timbiras, 1560, conj. 308 - Lourdes

Belo Horizonte/MG

CEP: 30.140-061

E-mail: silvia@ortoadultos.com.br 